\title{
DISTRIBUTION OF NETWORK GENERATED PROFIT BY CONSIDERING INDIVIDUAL PROFIT EXPECTATIONS
}

\author{
Hendrik Jähn' ${ }^{1}$, Marco Fischer ${ }^{2}$, Tobias Teich ${ }^{3}$ \\ ${ }^{\prime}$ Chemnitz University of Technology, hendrik.jaehn@wirtschaft.tu-chemnitz.de \\ ${ }^{2}$ Chemnitz University of Technology, marco.fischer@wirtschaft.tu-chemnitz.de \\ ${ }^{3}$ Zwickau University of Applied Sciences of West Saxony, tobias.teich@fh-zwickau.de
}

GERMANY

In this contribution approaches for the distribution of profit within networked production structures to the different network members are introduced and discussed. In this context exact rules are indispensable for the success of a cooperation because profits are the main target of all economic activities. In this context three influencing parameters are considered: a fixed share, a value-adding-dependent share and a profit expectation dependent share whereby the last mentioned parameter represents the most important variable.

\section{INTRODUCTORY REMARKS AND OVERVIEW}

In this contribution possibilities for the distribution of profit in production networks are focused. In that context it is assumed that a product is manufactured in a collaborative network of independent enterprises. The introduced models are of theoretical nature; however the practical relevancy is undisputed. In order to provide suitable solutions for different situations, several models have been elaborated. Basically it is assumed that a central operation and coordination instance operates as a broker for the whole network.

In existent literature hardly any comprehensive models focusing that topic can be identified. As an exception game-theoretic approaches can be identified (Fromen, 2005; Sucky, 2004) but there are no approaches taking into account the macroeconomic approach of the New Institutional Economics (Furubotn, 2005). Thereby, informational asymmetries among the network members, opportunistic behaviour, a limited rationality of actors and the tendency for individual maximisation of utility are the basic assumptions in that context.

The following profit distribution models consider mainly three influence parameters. In addition to a value-adding-independent (fixed) and a value-adding-dependent (variable) part, a profit expectation-dependent component is taken into consideration. The profit share of an enterprise which is determined by this influence factor is called $g_{i}^{p}$. The variable represents the profit expectation-dependent profit share of an enterprise. The individual profit expectation of an enterprise (with regard to the individual value-adding-share $w_{i}$ ) can be indicated as an amount $g_{i}{ }_{i}$ or a percentage of the value-adding-share $g^{e p}{ }_{i}$. It is stored in a central data base which is neither accessible to the network members nor to the broker instance. There is no "open book" strategy in the network.

In the further modelling three different influence components are taken into consideration. Thereby it has to be differentiated whether the product offer price of the network $P^{\text {offer }}$ (respectively the offer profit $G^{\text {offer }}$ ) correspond to the final sales price $P^{\text {sales }}$ (respectively the sales profit $P$ ) to the customer. Thus, several possibilities

Jähn, H., Fischer, M., Tcich, T., 2007, in IFIP International Federation for Information Processing, Volume 243, Establishing the Foundation of Collaborative Networks; eds. Camarinha-Matos, L., Afsarmanesh, H., Novais, P., Analide, C.; (Boston: Springer), pp. 337-344 
of component integration arise for the calculation of the profit shares. The following paragraph 3 will focus that structure. A further approach consists in the introduction of weightings for each component which is discussed in section 4 .

In principle, the calculation of the network profit calculated in the offer $\left(G^{\text {offer }}\right)$ is based on the individual profit expectations of the enterprises $g_{i}^{e}$ or $g^{e p}{ }_{i}$ as was stated before. However, it is imaginable that, after the value adding-process has been carried out, the distributed profit expectation-dependent profit share $G^{p}$ does not correspond to the complete profit $G$ that can be distributed. This so-called undistributed remaining profit $G^{u r}$ is negative if a too high amount has been distributed already whereas it is positive if a profit share remains and can be distributed. In both cases, measures need to be taken in order to exactly distribute the earned profit. Therefore, either a corresponding revaluation or devaluation by the help of a standardisation parameter or the consideration of the two remaining components are imaginable. While the first mentioned approach will be discussed in paragraph 3.1, the second option will be focussed in paragraph 3.2. For the less complicating case that the offer profit and the realised profit correspond to each other, two different approaches are discussed next in paragraph 2.1 and 2.2.

It has to be stressed that the different approaches for profit distribution presupposes a flat-hierarchical structure with an independent broker. The sensible data are stored centrally for the calculation of individual profit shares in an automated way using the modern information and communication technology.

\section{MODELS CONSIDERING NO REMAINING PROFIT}

\subsection{Enterprise-related profit expectation $g^{e p}{ }_{i}$ as a basis}

In the simplest case, the calculation of the offer price $P^{\text {offer }}$ is based on the individual value-adding-shares $w_{i}$ and the corresponding profit expectations $g^{e p}{ }_{i}$ an enterprise stated. If the offer including the suggested price is accepted by a customer, the goods are produced and finally finished and delivered. In the usual case, the customer then pays the agreed sales price $P^{\text {sales }}$ including the profit $G$, which is based on the individual profit expectations $g^{e p}{ }_{i}$ and thus corresponds to the offer price. The achieved profit $G$ can completely be distributed to the single enterprises in the network, no rest remains. The calculation of the enterprise-related profit shares $g_{i}^{p}$ is made by multiplying the enterprise-related profit expectation (in per cent) $g^{e p}{ }_{i}$ with the net value-adding of an enterprise $w_{i}$, cf. equation (1).

$$
g_{i}^{p}=g_{i}^{e p} \cdot w_{i}
$$

Thus, the enterprises are given their desired profit share without any reductions or supplements. Finally, the distributed (profit-expectation-dependent) profit $G^{P}$ results from the sum of those individual profit expectation-dependent profit shares $g_{i}{ }_{i}$ of all enterprises. This profit expectation-dependently distributed profit $G^{P}$ corresponds to the offer profit $G^{o f f e r}$ and thus, the complete profit $G$ was distributed. There is no remaining profit $G^{u r}$.

One problem of this procedure is the initial parameter "individual profit expectation" $g^{e p}$. The enterprises indicate this parameter independent from a certain value adding-process. However, there is the problem of intended incorrect information. For this reason, a parameter should be introduced which eliminates outliers and obviously wrong numbers. This parameter is called the average percentage of the 
expected profit $g^{e p d}$ and it is equal for all enterprises. Thereby, however, we often face the situation that the distributed profit does not necessarily correspond to the profit that can be distributed, anymore. This is also dependent on the calculation methodologies for the $g^{e p d}$. Thus, further distribution mechanisms need to be used which are also valid if a profit $G$, that can be distributed, was achieved which deviates from the offer profit $G^{\text {offer }}$. These mechanisms are introduced next.

\subsection{Enterprise-related average profit expectations $\boldsymbol{g}^{e p d}$ as a basis}

For calculating the (weighted) average profit $g^{\text {epd }}$ (measured in per cent of $W$ ), equation (2) can be applied. The individual percentage of the profit expectation $g^{e p}{ }_{i}$ therefore is weighted by the share of the individual value-adding-process $w_{i}$ of the complete value-adding-process $W$. Thereby, it has to be considered that this parameter is enterprise-independent.

$$
g^{e p d}=\frac{\sum_{i=1}^{n}\left(g_{i}^{e p} \cdot w_{i}\right)}{W}
$$

In the next step, it is now possible to ascertain the profit expectation-dependent profit share for every enterprise $g_{i}^{\prime \prime}$. This variable represents the most important component of the complete profit share of an enterprise $g_{i}$. It is valid:

$$
g_{i}^{p}=g^{e p d} \cdot w_{i}
$$

Finally, the complete (profit expectation-dependent) profit $G^{P}$, that has so far been paid out, results from the sum of those individual profit expectation-dependent profit shares $g_{i}^{p}$ of all enterprises. Summing up the profit shares per enterprise is necessary in order to determine the further procedure. Thus, a comparison between the already distributed profit $G^{P}$ and the profit after the value adding-process, that can in total be distributed, is the next step. By subtracting the already distributed profit from the distributable profit, a non-distributed remaining profit $G^{u r}$ results, which still can be distributed among the enterprises. It is valid:

$$
G^{u r}=G-G^{P}
$$

The total profit could be completely distributed based on the (non-weighted) profit expectation (per cent) $g^{e p}{ }_{i}$. When applying the profit expectation, that is weighted using the individual value adding-process, it has to be checked subsequently if this condition is fulfilled.

Here, it is significant to stress an effect of the application of the equations (2) and (3). This effect occurs in case the offer profit $G^{o f f e r}$ corresponds to the distributable profit $G$. This is not unusual because the offer profit $G^{\text {offer }}$ is already based on the indications concerning the profit expectation of the enterprises $g^{e p}{ }_{i}$. Starting from the enterprise-related percentage of the profit expectations $g^{e p}{ }_{i}$ (related to the enterprise-related net value-adding-process $w_{i}$ and unweighted), the profit expectation-dependent profit share $g_{i}^{p}$ is calculated as follows:

$$
g_{i}^{p}=g^{e p} \cdot w_{i}
$$


As opposed to that, equation (3) determined the profit expectation-dependent profit shares $g_{i}^{p}$ in dependence of the weighted average percentage of the profit expectation, see calculation in equation (2). This procedure usually leads to different values for $g_{i}{ }_{i}$ than equation (5). However, it can be remarked that the distributable profit $G$ will in any case need to be distributed among the enterprises without a remaining profit $G^{u r}$. This happens independently from the kind of calculation. This effect is especially interesting because thus it is made sure that this procedure provides usable results in a simple way despite of the improved modelling.

It is guaranteed that this approach does not provide any procedure-specific remaining profit. However, those procedure-specific remaining profits must not be changed with the remaining profits, which occur in case the distributable profit $G$ from the sales price $P^{\text {sales }}$ is higher than the offer profit $G^{\text {offer }}$ in the offer price $P^{\text {offer }}$. This will be the focus of the following paragraphs.

\section{MODELS CONSIDERING REMAINING PROFIT}

\subsection{Solution with a standardisation parameter}

In case the distributable profit $\mathrm{G}$ differs from the offer profit $G^{\text {offer }}$ the balance forms the remaining profit. That amount can be positive $\left(G>G^{\text {offer }}\right)$ or negative $\left(G<G^{\text {offer }}\right)$. In that case, a corresponding distribution mechanism needs to be applied for the non-distributed remaining profit $G^{u r}$. One possibility for that is the application of a standardisation parameter. With regard to a reference parameter, the individual profit shares $g_{i}$ are adapted based on a standardisation by the profit expectation dependent profit shares $g_{i}^{p}$.

In the following, the procedure will be described. It is assumed that a certain amount $G^{d}$ could be distributed which is based on the individual profit expectations. Here, it is of secondary importance whether the distribution took place based on the individual profit expectation $g^{e p}{ }_{i}$ or on the weighted profit expectation $g^{e p d}$, because it could be shown that the distributed profit is the same in each case.

However, a profit $G$ could be realised which exceeds the distributed profit $G^{d}$. This requires an alternative distribution approach. The suggested standardisation parameter is calculated by proportioning the total profit $G$ and the profit expectationdependent profit share $G^{P}$ that has already been distributed, cf. equation (6):

$$
\gamma=\frac{G}{G^{P}}
$$

Finally, the profit $g_{i}^{p}$, that has already been distributed, is multiplied with $\gamma$ for calculating the individual profit share of an enterprise $g_{i}$, cf. equation (7).

$$
g_{i}=\gamma \cdot g_{i}^{p}
$$

When calculating the model using concrete numbers, it strikes that the profit share of an enterprise of the complete profit corresponds to the value adding-share of an enterprise of the entire value adding-process.

\subsection{Distribution by means of a fixed and a variable profit share}

A further distribution variant results by the inclusion of fixed and variable profit shares in addition to the profit expectation-dependent profit share. This approach can again be applied for positive as well as negative remaining profits. The distribution 
of that remaining non-distributed profit $G^{u r}$, which was calculated using equation (4), is made subsequently by a fixed and a variable profit share. Thus, it is valid as follows for the fixed profit share:

$$
g_{i}^{f i x}=\alpha \cdot \frac{G^{u r}}{n}
$$

$G^{u r}$ hereby is divided by the number $n$ of active enterprises of the network and multiplied by the distribution parameter $\alpha$ which weights the fixed share. The remaining profit share is calculated as follows:

$$
g_{i}^{\mathrm{var}}=(1-\alpha) \cdot G^{u \prime r} \cdot \frac{w_{i}}{W}
$$

By applying those equations, a distribution of the profit $G$ to the enterprises that is based on three components is realised. The calculation of the complete profit share $g_{i}$ of an enterprise results by summing up the profit expectation-dependent profit share $g_{i}^{p}$, the fixed profit share $g^{f i x}{ }_{i}$ and the variable profit share $g^{\text {iar }}{ }_{i}$ as equation (10) illustrates:

$$
g_{i}=g_{i}^{p}+g_{i}^{f i x}+g_{i}^{\text {var }}
$$

It becomes clear that this model applies a distribution parameter $\alpha$. Several possibilities are imaginable for determining $\alpha$ (Jähn, 2005). That parameter is calculated as a share of the enterprise-related fixed share of the value addingprocess. Thereby, it seems to be probable that, after the distribution, the complete profit $G$ still has not been distributed. In that case the standardisation parameter is applied again.

Because on the one hand, the performance-oriented profit distribution is favoured, but on the other hand enterprise with a small value-adding share $w_{i}$ should not be disfavoured, the profit distribution based on three components and using a variable distribution parameter $\alpha_{i}$ is recommended in case the numbers are similar.

\subsection{Interim Conclusion}

The necessity of taking remaining profits into consideration predominantly arises when the offer price and the sales price (and thus offer profit and sales profit) do not correspond. It has to be remarked that the application of $\alpha$ is only obligatory in the second model. In principle, several $\alpha$ could be applied in this connection. Our example, however, is restricted to the application of an individualised distribution parameter. All the further models render $\alpha$ dispensable - a fact that is absolutely desirable because the (only) consideration of the enterprise-related profit expectation $g^{e}{ }_{i}$ promises a higher rate of being accepted by end-consumers. However this option will increase the complexity of the process.

\section{THREE-COMPONENT-APPROACH WITH WEIGHTINGS}

\subsection{Fundamentals}

So far it has been shown that the profit $G$ realised within a value-adding-process can be distributed to the enterprises within a network according to the enterprise-related profit expectations - no matter if weighted or not - in case this amount corresponds 
to the offer profit $G^{o f f e r}$ or even exceeds it. From that perspective, a distribution with regard to value-adding-dependent and value-adding-independent shares can be neglected. However, because it is aimed at a distribution that is based on several parameters, an alternative modelling, which meets this requirement, will be introduced in the following. Thereby the distributions are made according to the stated profit expectations $G_{i}{ }_{i}$ as well as to the number $n$ of participating enterprises in the network and the value-adding-share of an enterprise $w_{i}$.

\subsection{2 Selection of Weightings}

Here, weightings $v_{j}$ for the three components (distribution parameters) are introduced. The determination of the weightings can be made based on established procedures such as the trade-off-procedure (Eisenführ, 2005). According to their relevance, the distribution parameters are given a weighting. The sum of all the weights has to be 1 for standardisation reasons.

The (partial) distributable profit share that belongs to every component can be calculated by multiplying the profit, which must be distributed, $G$ with the corresponding weighting. After that, the several components are treated separately before finally the three calculated partial profit shares are summed up to the complete profit $g_{i}$ that an enterprise is entitled to. In the following, the single procedures are dealt with.

\subsection{Modelling}

By multiplying the weight $v_{l}$ with the profit $G$, that can be distributed, the profit share $G^{P e}$, that is possible according to the individual profit expectation, can be calculated using equation (11):

$$
G^{P e}=v_{1} \cdot G
$$

This calculated value for $G^{P e}$ is compared to the value for the sum of the profit expectations of the enterprises $G^{e}$. Thereby, it is not important whether the pure profit expectation of the enterprises in per cent $g^{e p}$ or the average (weighted) profit expectation in per cent $g^{\text {epd }}$ are used for the calculation. The paragraphs 2.1 and 2.2 already illustrated that the same value for $G^{e}$ results in both cases.

The ascertainment of the value comparison can lead to different alternatives of acting. In case the two values do not correspond to each other, which should be the rule, it has to be cleared up whether the calculatorily possible profit share $G^{P e}$ or the sum of the profit expectations $G^{e}$ are used as the basis of the further calculations. Due to the fact that the least value has been assumed as liable in the previous approaches, that means it was always assumed that the enterprises receive their expected profit (if the profit is sufficient), it is now assumed that only the calculatorily possible profit share $G^{P e}$ is distributed.

This results in the necessity to aim at an adaptation if the two values $G^{P e}$ and $G^{e}$ do not correspond. Therefore, the standardisation parameter $\gamma$ is applied again. Thereby, it is valid that $G^{P e}$ and $G^{e}$ are proportioned according to the following equation (12): 


$$
\gamma=\frac{G^{P e}}{G^{e}}
$$

The value for $\gamma$ calculated thereby is subsequently multiplied with the corresponding absolute profit expectations of the enterprises $g_{i}{ }_{i}$. As a result, the (corrected) profit expectation-dependent profit share can be calculated for every enterprise. It is valid:

$$
g_{i}^{p}=\gamma \cdot g_{i}^{e}
$$

If all the individual profit expectation-dependent profit shares $g_{i}^{p}$ are summed up (as the first component), the complete profit expectation-dependent profit share needs to result according to the weighting $G^{P e}$. For this interdependency confer equation (14):

$$
\sum g_{i}^{p}=G^{P e}
$$

Those coherences can be merged to one single equation as described in the following. If the term from equation (11) is put into the equation (12) for $G^{P e}$ and this is again put into equation (13) for $\gamma$, the following equation (15) for the calculation of the profit share-dependent profit share of an enterprise results for a specific weighting of the three components:

$$
g_{i}^{p}=v_{1} \cdot G \cdot \frac{g_{i}^{e}}{G^{e}}
$$

A further detailing of the components in the numerator of the fraction is not necessary because they need to be calculated in advance in order to be able to calculate $G^{e}$. Thereby, the calculation rules of paragraph 2.2 are applied.

In the following, the fixed profit share $g^{f i x}{ }_{i}$ that is dependent on the number of the enterprises participating in the network is considered as a further component. According to equation (8) it is valid:

$$
g_{i}^{f i x}=v_{2} \cdot \frac{G}{n}
$$

By multiplying the corresponding weighting $v_{2}$ with the profit $G$, that can be distributed, the partial profit is ascertained which can be distributed within the scope of the second component $n$ (number of totally engaged enterprises in the valueadding network).

Finally, the share of the net value-adding-process of one enterprise in the complete value adding-process has to be considered as a third component. Thereby, it can again be traced back to the already applied equation (9) which only needs to be slightly modified. It is valid:

$$
g_{i}^{\mathrm{var}}=v_{3} \cdot G \cdot \frac{w_{i}}{W}
$$

The profit, that can be distributed, $G$ is multiplied with the corresponding weighting $v_{3}$ as well as the corresponding value-adding share of an enterprise $w_{i}$ in the complete value-adding-process of the network $W$.

4.4 Aggregation of the Components

After having considered the single components, it presents itself to develop an equation which allows the calculation of a enterprise-specific profit share $g_{i}$ based 
on pre-defined initial parameters. Therefore, equation (18) can serve as the basis. Thus, it is valid as follows for the combination of the equations (15) until (17):

$$
g_{i}=v_{1} \cdot G \cdot \frac{g_{i}^{e}}{G^{e}}+v_{2} \cdot \frac{G}{n}+v_{3} \cdot G \cdot \frac{w_{i}}{W}
$$

Thereby, the required initial parameters are: the three weightings $v_{l}, v_{2}$ and $v_{3}$, the realised profit $G$ that can be distributed, the profit expectations of the enterprises in per cent $g^{e p}{ }_{i}$ as well as the net value-adding of the enterprises $w_{i}$.

The gross value-adding $W$ can again be calculated by summing up from the individual net value-addings $w_{i}$ of the enterprises. The profit expectation of an enterprise in per cent $g^{e p}{ }_{i}$ allows the calculation of the total amount of the profit expectation of an enterprise by multiplying that value with the net value-adding $w_{i}$. If those values are summed up, the sum of the individual profit expectations of the enterprises $G^{e}$ results.

Based on equation (18), a simplified equation for the calculation of individual profit shares of the enterprises of a network can be developed by factoring out $G$ and converting the equation. This leads to equation (19):

$$
g_{i}=G \cdot\left(\frac{v_{1} \cdot g_{i}^{e}}{G^{e}}+\frac{v_{2}}{n}+\frac{v_{3} \cdot w_{i}}{W}\right)
$$

It becomes clear that only the individual profit expectation $g_{i}^{e}\left(\right.$ or $g^{e p}$ ) as well as the individual value-adding $w_{i}$ need to be available as enterprise-specific influence parameters for the model. This makes allowance for the claim for transparency.

\section{CONCLUSION}

In this contribution different approaches for the distribution of profit shares under consideration of collaborative network structures are introduced. These models are applicable for the information-technically implementation into highly automated operator concepts for enterprise networks or virtual enterprises because especially the profits can be regarded as a motivation basis for the contractual performance of the network participants. Thereby it has to be considered that the striving for individual maximisation of utility and the tendency to maximise the utility of the entire networks are concurring targets which must be harmonised in the context of the New Institutional Economics.

\section{REFERENCES}

1. Eisenführ F. Rationales Entscheiden. 4. Auflage. Berlin, Heidelberg, New York: Springer, 2003.

2. Fromen B. Faire Aufteilung in Unternehmensnetzwerken. Wiesbaden: DUV, 2004.

3. Furubotn EG, Richter, R. Institutions and Economic Theory: The Contribution of the New Institutional Economics. 2nd ed. Ann Arbor: University of Michigan Press, 2005.

4. Jăhn H, Fischer M, Zimmermann M. An Approach for the Ascertainment of Profit Shares for Network Participants. In Collaborative Networks and their breeding Environments. Camarinha-Matos L.M. et al., eds. New York: Springer, 2005; 257-264.

5. Sucky E. Koordination in Supply Chains - Spieltheoretische Ansätze zur Ermittlung integrierter Bestell- und Produktionspolitiken. Wiesbaden: DUV, 2004. 